\title{
Detecting the Effect of Very Low Amounts of Penetrants in Lipid Bilayers Using Raman Spectroscopy
}

\author{
Robert J. Meier, ${ }^{* \dagger}$ Agnes Csiszár, and Erwin Klumpp \\ Institute Agrosphere, Research Centre Jülich, Germany, D-52428 Jülich \\ Received: March 14, 2006; In Final Form: August 8, 2006
}

\begin{abstract}
We show that, by the influence on the gauche content in the acyl chains, the effect of very low concentrations (order $10^{-2} \%$ ) of penetrants (here 2,4-dichlorophenol) in lipid bilayers (here dipalmitoyl-glycerophosphatidylcholine) can be experimentally seen in the Raman spectra. The observed effects suggest increased order and are in accordance with previous X-ray diffraction and calorimetric data. These findings show great promise for studying in situ the effect of low amounts of various small molecules on cell membranes using Raman spectroscopy.
\end{abstract}

\section{Introduction}

Membranes consist, in principle, of well-ordered (aligned) molecular chains. Apart from the transport of useful nutritional ingredients across the cell membrane, harmful components might be taken up by the membrane and affect its structure. ${ }^{1,2}$ Furthermore, there is a long-standing discussion on whether anaesthetics act by effecting the cell membrane. ${ }^{3}$ Thus, characterization tools that enable us to study the structure (ordering) of the membrane also provide information on the penetration of species into the membranes.

One of the techniques that has been explored in the past to study small molecules penetrating membrane structures is Raman spectroscopy. The vibrational spectra that are probed with Raman spectroscopy give information on both the membrane as well as on the penetrant. Usually, a pretty high (10\% and higher) concentration of penetrants is applied in order to see the effect in the Raman spectrum. ${ }^{4,5}$ This, however, is likely to be a concentration far much higher than that starting to exert serious physiological effects in a living organisms. For instance, whereas halothane was studied from a concentration of $0.3 \mathrm{~mol}$ halothane/L mol DPPC (dipalmitoyl-glycerophosphatidylcholine), it was mentioned that the halothane level for general anaesthesia was substantially lower. ${ }^{5}$ Lieb et al. ${ }^{6}$ attempted to measure the effect of clinical levels of general anaesthetics via the Raman spectra but found no noticeable effect. Therefore, a more sensitive approach is required to probe the possible effect of very low levels of penetrants in membranes.

\section{Results and Discussion}

Raman spectroscopy probes molecular vibrations. When small molecules are added to the membrane system, penetration of these molecules in the lipid bilayer can be expected to lead to adaptation of the conformation of the lipid alkyl chains and/or to changes of the spectrum corresponding to the polar headgroup. ${ }^{7}$ The effects of conformational changes on the Raman spectra of alkanes and alkyl chains has been well-studied. ${ }^{8,9}$

\footnotetext{
* Corresponding author.

† Permanent address: DSM Research, P.O. Box 18, NL-6160 MD Geleen.
}
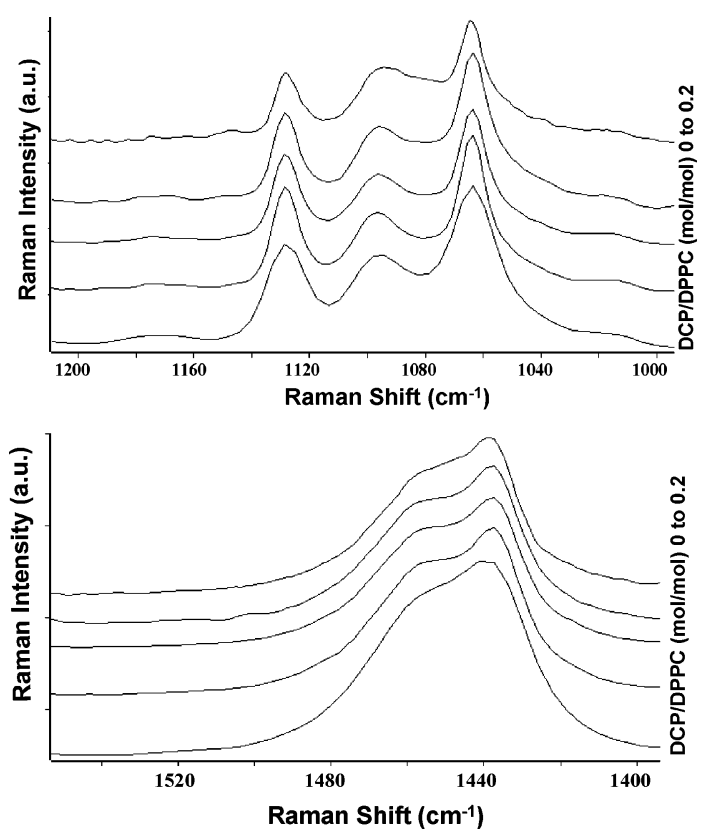

Figure 1. Top: Spectral range of $\mathrm{C}-\mathrm{C}$ stretching bands of DPPC and DPPC with DCP at the DCP/DPPC molar ratio of $0,4 \times 10^{-4}, 4$ $\times 10^{-2}, 0.1$, and 0.2 . Note that, within experimental error, the bandwidth for the 1060 and $1130 \mathrm{~cm}^{-1}$ bands is the same for the concentration range $4 \times 10^{-4}$ up to $4 \times 10^{-2}$. For further discussion, see text. Bottom: $\mathrm{CH}_{2}$ scissoring band of DPPC and DPPC with DCP at the DCP/DPPC molar ratio of $0,4 \times 10^{-4}, 4 \times 10^{-2}, 0.1$, and 0.2 . Spectra were recorded at $26^{\circ} \mathrm{C}$.

While studying the DCP/DPPC (2,4-dichlorophenol/dipalmitoyl-glycerophosphatidylcholine) system, ${ }^{7}$ we additionally noted small but clear differences in the Raman spectra at very low concentrations. Whereas we did not give this any particular attention beforehand, we have now become aware that this is a true phenomenon also revealed by other analytical techniques. Figure 1 reveals the Raman spectra in the $\mathrm{C}-\mathrm{C}$ stretching range (1060-1130 $\mathrm{cm}^{-1}$ ) and the $\mathrm{CH}_{2}$ scissoring range (around 1450 $\mathrm{cm}^{-1}$ ) for DCP concentrations ranging from $4 \times 10^{-4}$ up to 2 $\times 10^{-1} \mathrm{~mol} / \mathrm{mol}\left(4 \times 10^{-2}-20 \%\right)$. Note that there are clear differences between the spectra for $[\mathrm{DCP}]=0$ and $[\mathrm{DCP}]=4$ 


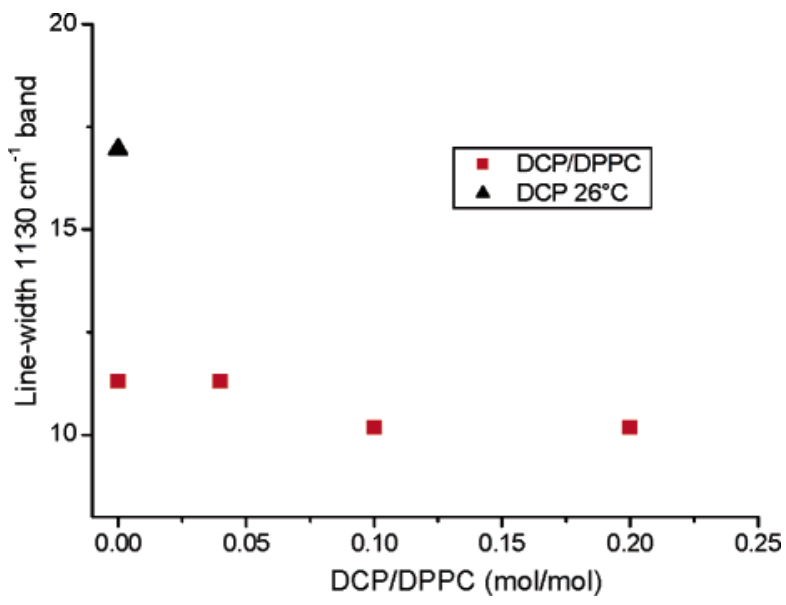

Figure 2. Raman line-width of the $1130 \mathrm{~cm}^{-1} \mathrm{C}-\mathrm{C}$ stretching band, derived from spectra recorded at $26^{\circ} \mathrm{C}$. Data points are for DCP/DPPC molar ratios of $0 / 100,0.04 / 100,4 / 100,10 / 100$, and 20/100. Note the significant drop in line-width when going from 0 to $0.04 \%$ DPC in DPPC.

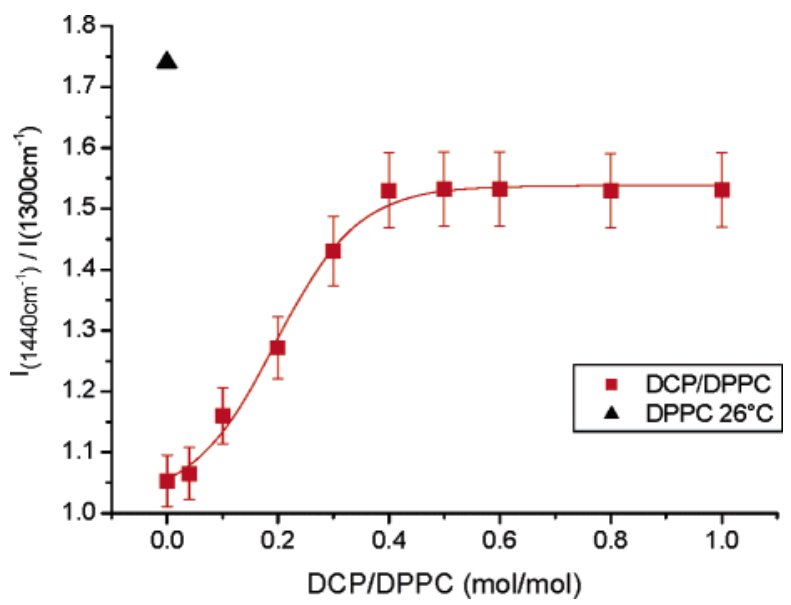

Figure 3. Intensity ratios of the $1440 \mathrm{~cm}^{-1}$ methylene scissoring /methylene twist modes of DPPC liposomes at the DCP/DPPC molar ratio of $0 / 100,0.04 / 100,4 / 100,10 / 100,20 / 100,30 / 100,40 / 100,50 / 100$, $60 / 100,80 / 100$, and $100 / 100$ at $26^{\circ} \mathrm{C}$. These ratios were obtained after curve fitting the band profile and then referencing the area versus the $1300 \mathrm{~cm}^{-1}$ area which can be taken as an internal standard. ${ }^{10,11}$ Please note the significant step when going from 0 to $0.04 \%$ DCP in DPPC.

$\times 10^{-4}$. Note that, within experimental error, the bandwidth for the 1060 and $1130 \mathrm{~cm}^{-1}$ bands is the same for the concentration range $4 \times 10^{-4}$ up to $4 \times 10^{-2}$. Figure 2 shows the line-width of the $1130 \mathrm{~cm}^{-1} \mathrm{C}-\mathrm{C}$ stretching band as a function of the ratio DCP/DPPC, and it is apparent that a significant change is observed when going from 0 to $0.04 \%$ DCP. Furthermore, the intensity of the $1440 \mathrm{~cm}^{-1}$ band, when referenced to the $1300 \mathrm{~cm}^{-1}$ range which can be taken as an internal standard, ${ }^{10,11}$ also exhibits a sudden change when going from 0 to $0.04 \%$ DCP as illustrated in Figure 3. Finally, the band centered near $1080 \mathrm{~cm}^{-1}$, which is due to disordered (gauche bonds) alkyl chains, broadens toward smaller Raman shifts with increasing DCP concentrations (DCP/DPPC ratio $=0.2)$. This is apparent from Figure 1, as the relative intensity of this band in the $1080 \mathrm{~cm}^{-1}$ range increases whereas the overlapping band centered at $1060 \mathrm{~cm}^{-1}$ narrows with increasing DCP concentration. This latter observation is consistent with the observation of a phase transition near a DCP/DPPC ratio of $0.1 .^{7}$

This observation is not accidental, as we have observed corresponding features in differential scanning calorimetry (DSC) curves ${ }^{12,13}$ and in small-angle X-ray scattering (SAXS) data. ${ }^{12}$ In the DSC curves, a small increase of the transition temperature was observed for the main transition as well as for the pre-transition. The SAXS data ${ }^{12}$ exhibit a higher and sharper Bragg peak for very low DCP concentrations compared to pure DPPC. This suggests an increased regularity or order for the low concentration DCP system. The evidence from SAXS and DSC further proves that the observations in our Raman spectra are not artifacts but the result of a true physical phenomenon. The detailed molecular interpretation remains further elucidation.

Although no special emphasis was put on these findings in our earlier reporting, , $^{12,13}$ the confirmation that the effects are also observable in the vibrational (Raman) spectra has drawn our attention. The observed very low concentration effects are likely to be potentially very relevant with respect to the study of biological membranes and the potential actions of penetrants, including soil contaminants originating from pesticides, anaesthetics, etc. As mentioned above, it has been a problem to study the effects of low concentration penetrants on the membrane structure, and the lack of Raman signal has led to the conclusion that clinical levels of anaesthesia do not involve the membrane structure. ${ }^{6}$

The observed effects in the DSC curves as well as in the SAXS curves suggest increased order when a very low concentration of the penetrant is present. The Raman spectra, more specifically the $\mathrm{C}-\mathrm{C}$ stretching bands in the $1060-1130$ $\mathrm{cm}^{-1}$ spectral range, reveal slight band narrowing after adding a very low concentration of DCP. This also, generally, points toward higher order. This is not unexpected, as experimental Raman spectra of pure DPPC reveal intensity in spectral regions corresponding to gauche bonds and, therefore, to some extent, disorder of the alkyl chains ${ }^{14,15}$ suggesting there is potential for an increase in order. This, as we have now observed, is indeed the case for very low levels of penetrants.

It is interesting, though still speculative, to think about whether the increased order at very low levels of penetrant is a characteristic property of biological membranes having a useful biological meaning. Future experiments are required to further elucidate this and other questions.

\section{References and Notes}

(1) Bicknell-Brown, E.; Brown, K. G. Biochim. Biophys. Acta 1984, 778, 317-323.

(2) Csiszar, A.; Klumpp, E.; Bota, A.; Szegedi, K. Chem. Phys. Lipids 2003, 126, 155-165

(3) Akeson, M.; Deamer, D. W. In Drug and Anaesthetic Effects on Membrane Structure and Function; Aloia, R. C., Curtain, C. C., Gordon, L. M., Eds.; Wiley-Liss: New York, 1991.

(4) Akutsu, H.; Suezaki, Y.; Yoshikawa, W.; Kyogoku, Y. Biochim. Biophys. Acta 1986, 854, 213. 2449.

(5) Craig, N. C.; Bryant, G. J.; Levin, I. W. Biochemistry 1987, 26,

(6) Lieb, W. R.; Kovalycsik, M.; Mendelsohn, R. Biochim. Biophys. Acta 1982, 388.

(7) Csiszár, A.; Koglin, E.; Meier, R. J.; Klumpp, E. Chem. Phys. Lipids 2006, 139, 115-124.

(8) Bower, D. I.; Maddams, W. F. The vibrational spectroscopy of polymers; Cambridge University Press: Cambridge, 1989.

(9) Painter, P. C.; Coleman, M. M.; Koenig, J. L. The Theory of Vibrational Spectroscopy and its Application to Polymeric Materials; John Wiley \& Sons: New York, 1982.

(10) Strobl, G. R.; Hagedorn, W. J. Polym. Sci., Polym. Phys. 1978, 16,1181

(11) Koglin, E.; Meier, R. J. Comput. Theor. Polym. Sci. 1999, 9, 327.

(12) Csiszár, A.; Bóta, A.; Novák, C.; Klumpp, E.; Subklew, G. Prog. Colloid Polym. Sci. 2001, 117, 145-152.

(13) Csiszár, A.; Bóta, A.; Novák, C.; Klumpp, E.; Subklew, G. J. Thermal Anal. Calorim. 2002, 69, 53-63.

(14) Meier, R. J.; Csiszár, A.; Klumpp, E. J. Phys. Chem. B 2006, 110, $5842-5844$

(15) Gaber, B. P.; Peticolas, W. L. Biochim. Biophys. Acta 1977, 465, $260-274$. 